\title{
Wunsch und Wirklichkeit
}

\author{
Frank Hinder, Hugo Van Aken
}

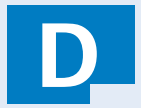

ie Sepsis ist ein häufiges Krankheitsbild auf der Intensivstation und stellt derzeit die Haupttodesursache auf nicht-kardiologischen Intensivstationen dar. Kürzlich wurden die ersten Daten der „Deutschen Prävalenzstudie“ veröffentlicht, die im Jahre 2003 durch das „Deutsche Kompetenz-Netzwerk Sepsis“ (SepNet) durchgeführt wurde [1]. Es wurden 3877 Patienten auf 454 randomisiert ausgesuchten deutschen Intensivstationen untersucht. 12,2\% der Patienten hatten eine Sepsis, weitere 10,7\% litten an einer Sepsis mit Organkomplikationen, der schweren Sepsis und dem septischen Schock.

Die Letalität von Patienten mit schwerer Sepsis bzw. septischem Schock betrug auf der Intensivstation $47 \%$, im Krankenhaus 54\% [1]. Angesichts dieser Zahlen erscheint es dringend geboten, evidenzbasierte Therapieansätze konsequent in die klinische Praxis zu implementieren. Was zunächst banal klingt, ist durchaus nicht selbstverständlich. Im Rahmen der SepNet-Prävalenzstudie zeigte sich eine klaffende Lücke zwischen Wunsch und Wirklichkeit, zwischen der Einschätzung der Verantwortlichen der Intensivstationen, in welchem Maße konsentierte Therapiestandards auf ihren Intensivstationen umgesetzt werden, und der Realität auf der Station am Tage der Evaluation [2]. 91,6\% der verantwortlichen Intensivmediziner waren der Meinung, auf ihrer Intensivstation würden Patienten mit akutem ALI/ARDS lungenprotektiv beatmet (TV: $6 \mathrm{ml} /$ $\mathrm{kg} /$ korrigiertes Körpergewicht). Dies war aber nur in 4,2\% der Patienten der Fall. 79\% der Intensivmediziner gaben an, bei septischem Schock niedrig dosiertes Hydrokortison zu verwenden, aber nur 30,6\% der Patienten mit septischem Schock erhielten das Medikament. Umgekehrt wurde in $23 \%$ der Patienten mit schwerer Sepsis eine Hydrokortison-Therapie durchgeführt, ohne dass es dafür eine wissenschaftliche Evidenz gibt. Die beschriebene Diskrepanz zwischen Einschätzung und Realität in der Anwendung evidenzbasierter Therapieansätze ist keine auf die Sepsistherapie beschränkte Beobachtung. Eine Studie zur Behandlung von Patienten mit Linksherzinsuffizienz nach Myokardinfarkt zeigte, dass weniger als 20\% der Patienten Angiotensin Converting Enzyme (ACE)-Inhibitoren erhielten, selbst wenn die Patienten an einem
Krankenhaus behandelt wurden, das an der SAVE-Studie (Survival and Ventricular Enlargment Trial) teilgenommen hatte, die Vorteile der Therapie mit ACE-Inhibitor hinsichtlich Überleben und Ventrikelgröße in diesem Patientenkollektiv ergeben hatte [3]. Cook und Kollegen analysierten, warum Empfehlungen zur Prävention beatmungsassoziierter Pneumonien in der Intensivmedizin nicht umgesetzt wurden [4]. Als Gründe wurden u.a. eine mangelnde Übereinstimmung mit der Interpretation klinischer Studien, unzureichende Ressourcen, die Annahme, die Maßnahmen könnten Nebenwirkungen haben, und Kosten spezifischer Interventionen angeführt. Sicher sind sowohl eine kontinuierliche Fortbildung wie auch schriftlich fixierte Verfahrensanweisungen notwendig bei dem Versuch, die Therapie zu standardisieren, aber eben nicht hinreichend. In der Industrie ist seit Jahrzehnten der von William E. Deming, dem US-amerikanischen Pionier der Qualitätssicherung, entwickelte Plan-Do-CheckAct-Zyklus (PTCA-Zyklus) ein häufig eingesetztes Instrument zum Erreichen der Anforderungen des Qualitätsmanagements. Die Schritte „Check“, die Überprüfung des Erreichten, und „Act“, worunter man die Konsequenzen im weitesten Sinne verstehen kann, die aus dem Ergebnis der Qualitätsprüfung gezogen werden, sind „conditiones sine qua non“ in einem Verbesserungsprozess. Das vielfach bewährte PTCAKonzept liegt nun einem internationalen Projekt der Surviving Sepsis Campaign zugrunde, mit dem die Prognose von Patienten mit Sepsis verbessert werden soll.

Die Surviving Sepsis Campaign wurde 2002 als Kooperationsprojekt der European Society of Intensive Care Medicine, des International Sepsis Forum und der Society of Critical Care Medicine ins Leben gerufen. Man hatte sich zusammengefunden, um sich gemeinsam den Herausforderungen der Sepsis zu stellen, auf nationaler und internationaler Ebene ein stärkeres Bewusstsein für dieses Krankheitsbild zu schaffen und gemeinsame Strategien für die Diagnose und Therapie der Sepsis voranzubringen.

Das ehrgeizige Ziel der Surviving Sepsis Campaign ist, die Letalität von Patienten mit Sepsis innerhalb von fünf Jahren um $25 \%$ zu reduzieren. Als vordringlichste 
Aufgabe wird dabei angesehen, das Verhalten der Therapeuten dahingehend zu fördern, dass das Krankheitsbild noch schneller identifiziert wird und zuvor konsentierte Therapiealgorithmen zuverlässig und zeitnah umgesetzt werden. In der „Deklaration von Barcelona“ wurden diese Ziele in 2002 verabschiedet.

Es wurden evidenzbasierte Empfehlungen für die Diagnose und Behandlung der Sepsis, die „Surviving Sepsis Campaign Guidelines for the Management of Severe Sepsis and Septic Shock“ im Frühjahr 2004 publiziert [5]. Mittlerweile war es gelungen, 11 medizinische Gesellschaften, deren Mitglieder an der Behandlung septischer Patienten beteiligt sind, für die gemeinsame Anstrengung zu gewinnen. Auch die Deutsche Sepsis-Gesellschaft hat in Zusammenarbeit mit der Deutschen Interdisziplinären Vereinigung für Intensiv- und Notfallmedizin (DIVI) Leitlinien für die Diagnostik und Therapie der Sepsis publiziert [6], die als Weiterentwicklung der Surviving Sepsis Campaign Guidelines anzusehen sind.

Für die Surviving Sepsis Campaign dürfte aktuell der schwierigste Teil des Vorhabens, die Letalität der Sepsis zu reduzieren, begonnen haben; nämlich die Implementierung der konsentierten Empfehlungen in die tägliche Praxis und die Evaluation der Umsetzung. Hierbei sollen empfehlungsbasierte, sogenannte Severe Sepsis Bundles in die Behandlung der Patienten integriert und die Güte der Umsetzung täglich durch die Therapeuten selbst evaluiert werden. Bei den Severe Sepsis Bundles handelt es sich um zwei Bündel spezifischer Maßnahmen zur Diagnostik und Therapie der Sepsis, das Sepsis Resuscitation Bundle und das Sepsis Management Bundle. Diese Maßnahmen beruhen auf den Surviving Sepsis Campaign Guidelines, werden aber, insbesondere im Hinblick z. B. auf die Gabe von Medikamenten wie dem aktivierten Protein $\mathrm{C}$, an die Gepflogenheiten eines individuellen Krankenhauses angepasst. Das Sepsis Resuscitation Bundle beinhaltet Maßnahmen, deren Umsetzung innerhalb von sechs Stunden bewertet wird. Dazu zählen die Bestimmung des Serumlaktatspiegels und eine frühzeitige zielgerichtete Herzkreislauftherapie in Anlehnung an die Vorgaben der vielzitierten Rivers-Studie [7], aber auch die Abnahme von Blutkulturen und der sich anschließende Beginn einer kalkulierten Breitbandantibiose innerhalb von einer Stunde nach Diagnose der Sepsis. Die Umsetzung der an den Standards des Krankenhauses orientierten Vorgaben im Sepsis Management Bundle, zu denen die niedrigdosierte Hydrokortisontherapie, die Gabe von aktiviertem Drotregogin alpha, die Einstellung des Blutglukosespiegels und die Be- grenzung des Plateaudruckes bei beatmeten Patienten gehören, wird nach 24 Stunden bewertet. Die Bewertung und Selbstkontrolle erfolgt anhand eines Computerprogramms, über das die Daten auch anonymisiert in eine internationale Datenbank übermittelt werden. Dies ermöglicht dem Intensivteam, regelmäßig die Qualität der eigenen Arbeit mit der aller übrigen teilnehmenden Zentren zu vergleichen. Möge dieses Projekt, das die Selbstkontrolle der Qualität auf unseren Intensivstationen fördern wird, Schule machen und die Wirklichkeit dem Wunsch, unseren Patienten die bestmögliche Therapie zukommen zu lassen, ein Stück näher bringen. Wir wünschen dem Projekt viel Erfolg.

\section{Weiterführende Literatur}

1 Brunkhorst FM, Engel C, Bone H-G, et al. Epidemiology of severe sepsis and septic shock in Germany - Results from the German Prevalence Study. Infection 2005; 33 (Suppl. 1): 49

2 Brunkhorst FM, Engel C, Jaschinsky U, et al. Treatment of severe sepsis and septic shock in Germany: the gap between perception and practice - Results from the German Prevalence Study. Infection 2005; 33 (Suppl. 1): A112

3 Majumdar SR, Chang WC, Armstrong PW. Do the investigative sites that take part in a positive clinical trial translate that evidence into practice? Am J Med 2002; 113: 140 - 145

4 Cook D, Ricard JD, Reeve B, et al. Ventilator circuit and secretion management strategies: a Franco-Canadian survey. Crit Care Med 2000; 28: 3547 - 3554

5 Dellinger RP, Carlet JM, Masur H, et al. Surviving Sepsis Campaign guidelines for management of severe sepsis and septic shock. Intensive Care Med 2004; 30: 536- 555

6 Deutsche Sepsis-Gesellschaft e.V., Deutsche Interdisziplinäre Vereinigung für Intensiv- und Notfallmedizin. Diagnose und Therapie der Sepsis. 2005 http://www.awmf-online.de.

7 Rivers E, Nguyen B, Havstad S, et al. Early goaldirected therapy in the treatment of severe sepsis and septic shock. N Engl J Med 2001; 345: $1368-1377$

\section{Korrespondenzadresse}

Prof. Dr. med. Frank Hinder

Klinik und Poliklinik für Anästhesiologie und operative Intensivmedizin, Universitätsklinikum Münster

Albert-Schweitzer-Str. 33, 48149 Münster

Telefon: 0251/8347255

E-mail: hinder-f@anit.uni-muenster.de 